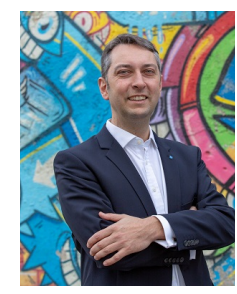

\section{Sebastian Schlund}

Institutsvorstand

am Institut für Managementwissenschaften, BMK-Stiftungsprofessor, Technische Universität Wien

sebastian.schlund@tuwien.ac.at

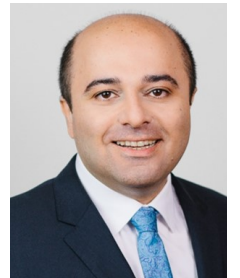

\section{Fazel Ansari}

Privatdozent und Forschungsgruppenleiter am Institut für Managementwissenschaften, Leiter der Forschungsgruppe Smart and Knowledge Based Maintenance, Technische Universität Wien

fazel.ansari@tuwien.ac.at

\title{
Fit for Future Work
}

\section{Dynamisierung des Kompetenz-Profiling mithilfe KI-gestützter Datenanalyse betrieblicher Prozesse}

Der Mangel an digitalen Kompetenzen wird von Unternehmen häufig als eine große, wenn nicht gar als größte ${ }^{1}$, Barriere auf dem Weg der digitalen Transformation gesehen. Gleichzeitig verfolgen die meisten Unternehmen keine übergeordnete Strategie zur Erhöhung ihrer Kompetenzen im Zuge der aktuellen Integration von Digitalisierungs- und Automatisierungstechnologien ${ }^{2}$. Ohne die valide Abschätzung erforderlicher zukünftiger Kompetenzen der Mitarbeiterlnnen in technischen Bereichen, sind zielgerichtete Entwicklungsmaßnahmen nicht möglich, was wiederum zu einer weiteren Vergrößerung der Kompetenzlücken in Unternehmen führt. Durch die zielgerichtete, KI-gestützte Nutzung bereits bestehender Daten aus organisationsinternen und -externen Quellen lassen sich Kompetenzmodelle dynamisieren und Unternehmensdaten der tatsächlichen Kompetenznutzung bei der Lösung betrieblicher Probleme integrieren.

\section{Kompetenzmanagement als unternehmerische Gestaltungsaufgabe}

Das betriebliche Kompetenzmanagement stellt eine unternehmensinterne Reaktion auf externe und interne Veränderungen der erforderlichen Fähigkeiten und Fertigkeiten dar. Neben der Analyse und Entwicklung bestehender Kompetenzen, Fertigkeiten und Qualifizierungspfade gilt es hier, mit Entwicklungen umzugehen, denen ein hohes Disruptionspotenzial zugeschrieben wird. Gleichzeitig stehen Unternehmen zunehmend unter Druck, technologische Entwicklungen aufzunehmen und in ihre Produkte, Services und Prozesse zu integrieren. Während der letzten Jahre wurde dieses Phänomen sehr gut sichtbar bei der Nutzbarmachung der Digitalisierung, Automatisierung und Intelligentisierung in (Industrie-)Unternehmen. Ausgelöst wurde dies durch Innovationssprünge im Bereich der wirtschaftlichen Nutzung von Technologien, wie der Robotik, Künstlicher Intelligenz (KI) oder Additiver Fertigung. Eine Vielzahl der Unternehmen 
wählten erfolgreich den Weg der Einführung in betrieblichen Pilotbereichen. Weniger erfolgreich vollzieht sich häufig jedoch die betriebliche Skalierung und somit zuletzt auch die wirtschaftliche Technologienutzung. Ein häufig genanntes Hemmnis dafür ist die fehlende Qualifikation der Mitarbeiterlnnen ${ }^{3}$.

Organisationales Kompetenzmanagement ${ }^{4,5}$ beschreibt die Umsetzung strategischer Ziele und Veränderungsprozesse durch die Verknüpfung von organisationalem Lernen, Gruppenlernen und individuellem Lernen. Grundlage dafür sind i.d.R. ein Kompetenzmanagementsystem, ein betriebliches Kompetenzmodell, die Möglichkeit individueller Messungen bzw. Kompetenzeinschätzungen sowie auf das Kompetenzmodell ausgerichtete Personalinstrumente. Obwohl meist in den Personalbereichen (Human Ressources) verortet, bedingt die Orientierung einer Organisation am Kompetenzmanagement die durchgängige und intensive Einbindung der jeweiligen Fachabteilungen. Das Kompetenzmodell als zentrales Element des betrieblichen Kompetenzmanagements ordnet die relevanten Aufgaben (Tasks) den dafür notwendigen Fähigkeiten, Fertigkeiten und Kompetenzen (Competencies, Skills, Abilities) der MitarbeiterInnen zu. Da diese Zusammenhänge neben der Darstellung der heutigen Kompetenzen vor allem die zukünftige Gestaltung der Kompetenzentwicklung betreffen, beinhaltet ein jedes Kompetenzmodell externe Unschärfen ${ }^{6}$. Diese treten aufgrund risikobehafteter Annahmen über die zukünftige Entwicklung der benötigten Aufgaben auf. Zudem kommt häufig eine interne Unschärfe hinzu, die durch Annahmen und Zusammenfassungen der tatsächlich zur operativen Ausführung betrieblicher Prozesse notwendigen Tätigkeiten zu verallgemeinerten und, im Sinne einer Personalentwicklung auch ohne die konkrete Anwendung, planbaren Kompetenzen entsteht.

Die Aufstellung des initialen Kompetenzmodells orientiert sich häufig an übergeordneten Rahmenmodellen bzw. Kompetenz-Frameworks. Auf europäischer Ebene hat sich der Europäische Qualifikationsrahmen (EQF) etabliert, der eine Vergleichbarkeit beruflicher Qualifikationen, v.a. Abschlüsse zum Ziel hat und in zahlreiche nationale Qualifikationsrahmen umgesetzt wurde. Eine Ebene tiefer bietet die europäische Klassifikation für Fähigkeiten, Kompetenzen, Qualifikationen und Berufe (ESCO) eine Sammlung von mehreren tausend kompetenzrelevanten Artefakten und somit einen Referenzrahmen für ihre Beschreibung. Für die Beherrschung externer Unschärfen bei der Aktualisierung des Kompetenzmodells werden heute häufig zwei Ansätze kombiniert.

Zum einen orientiert sich die Erwartung zukünftig benötigter Kompetenzen an einer Extrapolation der heute benötigten. Zum anderen werden im Sinne eines Technologiemanagement-Ansatzes heute sichtbare Signale berücksichtigt, die in den nächsten Jahren die betrieblichen Prozesse und somit auch die benötigten Kompetenzen beeinflussen werden. Beide Ansätze bieten ein hohes Potenzial für die Nutzung von Methoden aus dem Bereich der KI; hier insbesondere aus dem Bereich des NLP (Natural Language Processing) und semantischer Technologien, vor allem Text Mining Methoden und 
semantische Modelle (Taxonomie, Ontologie, etc. $)^{6}$. Im vorliegenden Beitrag steht die Nutzung von Text Mining-Methoden und semantischer Modelle für das Kompetenz-Profiling auf der Basis großer Unternehmensdatensätze im Vordergrund. Für den zweiten Aspekt der KI-gestützten Analyse von Trends und deren (teil-)automatisierte Bewertung als Eingangsgröße für das Kompetenzmodell sei auf Abbildung 1 verwiesen.

\section{KI-gestütztes Kompetenz-Profiling}

Text Mining „(auch Text Data Mining oder Textual Data Mining) umfasst statistische und linguistische Ansätze zur maschinellen Erschließung unbekannter Informationen aus schwach oder nicht strukturierten Ressourcen“7. Die Grundidee der Nutzung von Text Mining-Methoden ${ }^{8}$ für die automatisierte Auswertung großer betrieblicher un- und teilstrukturierte Datensätze ist die Ausgangshypothese, dass die Aktualisierung eines Kompetenzmodells durch die genaue Analyse tatsächlich aufgetretener Problemlösungssituationen verbessert werden kann. Für diese liegen i.d.R. in Unternehmen Dokumentationen vor, die je nach Anwendungsbereich und Unternehmensspezifika unoder teilstrukturiert sind. Diese Datensätze können nunmehr hinsichtlich ihres Inhalts durchsucht und anhand zielrelevanter Attribute strukturiert werden. So kann ein Instandhaltungsbericht beispielsweise hinsichtlich der genutzten Nomen und Verben sowie der jeweiligen Wortfrequenzen untersucht werden ${ }^{8}$. Mithilfe von Tätigkeitsbeschreibungen als Verb-Nomen-Kombinationen (bspw. „Komponente XY austauschen“) sowie einer semantischen und maschinenlesbaren Zuordnung zu einer Kompetenztaxonomie können nunmehr kompetenzrelevante Ereignisse anhand real aufgetretener Situationen ausgewertet werden. Neben der qualitativen Analyse erlaubt die Nutzung großer Datensätze hierbei auch quantitative Rückschlüsse, bspw. über den zeitlichen Verlauf der aufgetretenen Ereignisse, sowie Zuordnungen zu einzelnen Ursachen und Personen.

Abbildung 1 zeigt exemplarisch ein Kompetenzmodell und dort im oberen Bereich das zentrale Ziel des Abgleichs der Bedarfsinformationen aus den Fachbereichen mit den angeforderten Kompetenzen und Qualifikationen der Personalplanung. Im unteren Teil der Abbildung sind diese beiden Seiten des Kompetenzmodells aufgegliedert. Als Informationsgrundlage der Personalplanung werden definierte Jobprofile mitsamt der jeweils benötigten Kompetenzen und deren Ausprägungs-Soll-Werten definiert. Diese beinhalten Einzelfähigkeiten (Sub-Kompetenzen), wie beispielsweise Fähigkeiten zur Problemlösung, Kommunikation und technische Kompetenzen unterschiedlicher Fachdisziplinen. Seitens der Bedarfsinformationen aus den Fachbereichen (rechte Seite der Abbildung) wird für das Kompetenzmatching dargestellt, welche Kompetenzen durch die tatsächlich auftretenden Aufgaben benötigt werden. Dazu werden vorliegende Daten (Berichte) mit Text-Mining-Methoden hinsichtlich der verwendeten Nomen und Verben ausgewertet und kategorisiert (bspw. nach Aufgaben, Tätigkeiten und Auslösern/Ursachen). Im Anschluss erfolgt eine Überführung der Ursache/Tätigkeits- 
Kombinationen in die benötigten Kompetenzen samt ihrer Ausprägungen (hier für den Instandhaltungs-Shopfloor (SF)). Durch den Vergleich der beiden Kompetenzsets können Abweichungen, Kompetenzlücken und Trends identifiziert und darauf aufbauend Handlungsmaßnahmen definiert werden.

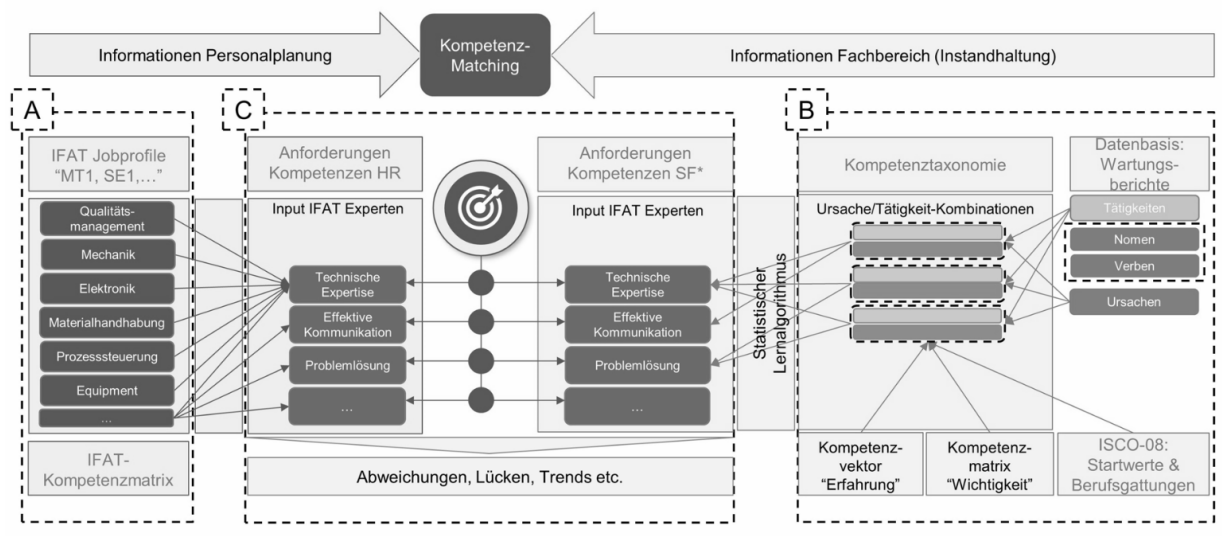

Abbildung 1: Kompetenzmodell und Kompetenzmatching ${ }^{9}$

Ein interdisziplinäres Projektteam, bestehend aus Vertretern der operativen Bereiche, etwaiger Supportbereiche und der HR, sammelt und bewertet bestehende Prozesse und Tätigkeiten. Des Weiteren werden bestehende Bereichsdaten (bspw. Instandhaltungsdokumentationen, Prozessdaten, etc.) gesammelt und mithilfe von Text-Mining-Methoden ausgewertet. Die Analyse und Bewertung relevanter Technologietrends in Verbindung mit der Auswertung global verfügbarer Web-Daten erlaubt die Ableitung zukünftiger Rollen, Aufgaben und Kompetenzen. Diese werden anschließend in ein Rahmenmodell zur Planung der Mitarbeiterentwicklung überführt.

\section{Umsetzungsbeispiel: Vom Wartungsbericht zur Kompetenzlücke}

Zusammen mit der Infineon Technologies AG Österreich wurde im Zeitraum von August bis Dezember 2020 das Projekt TEXCOM ${ }^{10}$ „Text-Mining gestütztes Kompetenz-Profiling" für einen Pilotbereich der Instandhaltung am Standort in Villach durchgeführt. Als Ausgangssituation standen folgende Bedarfe im Vordergrund:

- Bedarf eines instandhaltungsspezifischen Kompetenzmodells zur Optimierung von Personalplanung und -management

- Bedarf, Kompetenzlücken zwischen vorhandenen und tatsächlich erforderlichen technischen, Problemlösungs- und Kommunikationskompetenzen zu schließen.

- Bedarf der Operationalisierung unstrukturierter Daten, insbesondere umfangreicher textbasierter Wartungsdokumentationen 
Für die Schaffung einer auswertbaren Datenbasis wurden Auszüge der Instandhaltungsdokumentation der letzten Jahre zusammengestellt. Im Anschluss daran konnten durch die Anwendung von Text Mining-Algorithmen und Pre-Processing kompetenzrelevante Artefakte extrahiert und analysiert werden. Auf deren Grundlage sowie unter Zuhilfenahme domänenspezifischer Erfahrung und bestehender Kompetenzkataloge wurde eine anwendungsbezogene Kompetenztaxonomie aufgebaut (siehe Abb. 2). Durch die Anwendung von Lernalgorithmen konnte die Taxonomie zu einem Kompetenzmodell für den Betrachtungsbereich weiterentwickelt und verallgemeinert werden. Neu entstehende Daten erweitern und verbessern dieses Modell und erhöhen die Aussagequalität.

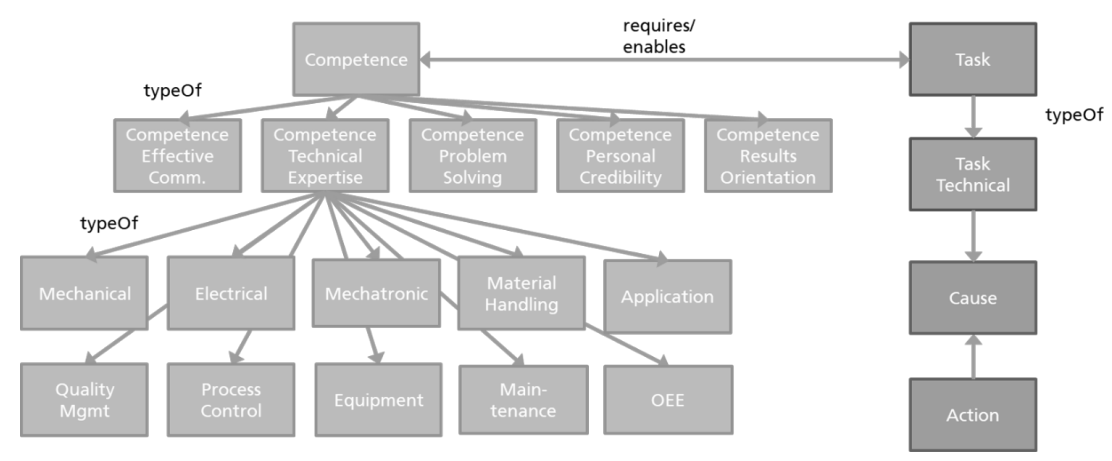

Abbildung 2: Kompetenztaxonomie als semantisches und maschinenlesbares Modell (Auszug) ${ }^{9}$

Im Ergebnis des Projekts stehen qualitative und quantitative Ableitungen von Kompetenzen aus Wartungstätigkeiten sowie deren Abgleich mit Jobanforderungen zur Verfügung. Auf dieser Grundlage können datenbasierte Tätigkeits- und Kompetenz-Trendanalysen durchgeführt werden. Diese spiegeln die Entwicklung von einzelnen Tätigkeiten und Tätigkeitsgruppen bestimmter Jobprofile genauso wieder wie die Verschiebungen der Tätigkeitsanteile (bspw. zwischen technischen und Problemlösungskompetenzen). Auf dieser Grundlage können nun datengestützt Handlungsempfehlungen zur Nutzung der Kompetenzabweichungen abgeleitet werden, welche die traditionellen Technologiemanagement-getriebenen Ansätze unterstützen, validieren bzw. auf mögliche Inkonsistenzen hinweisen. Neben der strategischen Nutzung zur Personalentwicklung erlauben die Daten und Erkenntnisse eine zielgerichtetere Planung von Personaleinsatz und -steuerung. Ein weiterer positiver Nebeneffekt des Projekts war eine Verbesserung der Dokumentation im Sinne entwickelter Richtlinien zur Erhöhung der Datenqualität bei der Eingabe der Dokumentationstexte. 


\section{Fazit und Ausblick}

Der Beitrag zeigt Nutzungsmöglichkeiten von Methoden von KI für die Verbesserung und Weiterentwicklung des betrieblichen Kompetenzmanagements. Insbesondere wird die Erweiterung des häufig bestehenden technologiemanagementgetriebenen Ansatzes um ein datenbasiertes System thematisiert und am Beispiel eines Umsetzungsprojekts erläutert. Für die Zukunft bleibt zu erwarten, in welchem Maße Unternehmen ihre vorhandenen und potenziellen Datenquellen verfügbar machen, auswerten und nutzen. Erfahrungsgemäß besteht in der initialen Erzeugung einer verarbeitbaren Datenqualität eine der aktuellen Hauptherausforderungen. Ist diese erst einmal erzeugt, bietet das Instrumentarium des Natural Language Processings sowie semantische Technologien ein vielfältiges und mächtiges Instrumentarium, um viel dynamischer und präziser auf unternehmensinterne und -externe Einflüsse und kompetenzrelevante Veränderungen reagieren zu können und somit einen Wettbewerbsvorteil im Kampf um die passfähigsten Fachkräfte zu erlangen.

\section{Literatur}

1 Schuh, G., Kelzenberg, C., Helbig, J., \& Graberg, T. (2020). Kompetenzprofile in einer digital vernetzten Produktion: Veränderungen in Kompetenzanforderungen und Handlungsempfehlungen für eine anforderungsgerechte Weiterbildung in der metallverarbeitenden Industrie, Working Paper Forschungsförderung, No. 198, Hans-Böckler-Stiftung, Düsseldorf.

2 Schumacher, A. \& Sihn, W. (2019). A Monitoring System for Implementation of Industrial Digitalization and Automation using 143 Key Performance Indicators.

3 Schlund, S. \& Pokorni, B. (2016). Industrie 4.0-Wo steht die Revolution der Arbeitsgestaltung. Ulm Stuttgart.

4 Grote, S., Kauffeld, S., \& Frieling, E. (Eds.). (2006). Kompetenzmanagement. Schäffer-Poeschel Verlag.

5 Heyse, V., \& Erpenbeck, J. (2009). Kompetenztraining. Informations- und Trainingsprogramme.

Stuttgart.

6 Ansari, F. (2019), Knowledge Management 4.0: Theoretical and Practical Considerations in Cyber Physical Production Systems,9th IFAC Conference on Manufacturing Modelling, Management and Control, Berlin, 2830 August, IFAC-PapersOnLine, 52(13): 1597-1602.

7 Enzyklopaedie der Wirtschaftsinformatik, Link: https://www.enzyklopaedie-der-wirtschaftsinformatik.de/lexikon/technologien-methoden/text-mining

8 Ansari, F. (2020). Cost-based text understanding to improve maintenance knowledge intelligence in manufacturing enterprises. Computers \& Industrial Engineering, 141: 106319.

9 Kohl, L., Fuchs, B., Berndt, R., Valtiner, D., Ansari, F., \& Schlund, S. (2021). Künstliche Intelligenz im Kompetenzmanagement. Zeitschrift für wirtschaftlichen Fabrikbetrieb, 116(7-8): 534-537.

$10 \mathrm{https}$ ///www.fraunhofer.at/de/zusammenarbeit/advanced-industrial-management/digitale-lern--und-informationssysteme/referenzen/texcom.html 


\section{Information zu den Autoren}

Univ.-Prof. Dr.-Ing. Sebastian Schlund ist BMK-Stiftungsprofessor für Industrie 4.0 an der Technischen Universität Wien und leitet dort den Forschungsbereich Mensch-Maschine-Interaktion am Institut für Managementwissenschaften (IMW). Seit dem 01.07.2019 verantwortet er zusätzlich den Geschäftsbereich „Advanced Industrial Management" bei der Fraunhofer Austria Research GmbH. Er forscht und lehrt im Themenbereich digital und automatisiert unterstützter Arbeitsgestaltung in der Produktion mit den Schwerpunktthemen Assistenzsysteme, Arbeitsorganisation, Mensch-MaschinePartnership, Montageplanung und Kompetenzentwicklung/Lernen.

Priv.-Doz. Dr.-Ing. Fazel Ansari ist Leiter der Forschungsgruppe „Smart and KnowledgeBased Maintenance" an der TU Wien. Sein interdisziplinärer Hintergrund wird durch ein Mechatronik-Studium und eine Dissertation in Informatik (Summa cum laude) an der Universität Siegen sowie seine Habilitation in Industrial Engineering an der TU Wien unterstrichen. Seit 2017 ist PD Dr.-Ing. Ansari an der TU Wien sowie als Senior Researcher und Projektleiter im Themenbereich digitale Lernen und Informationssysteme in der Fraunhofer Austria Research GmbH tätig. Er forscht und lehrt im Themenbereich Wissensmanagement 4.0, Instandhaltungsmanagement und cyber-physische Produktionssysteme. 